\section{A recommendation for training in stereotactic radiosurgery for US neurosurgery residents}

\author{
L. Dade Lunsford, M.D., ${ }^{1}$ Veronica Chiang, M.D., ${ }^{2}$ \\ John R. Adler, M.D., ${ }^{3}$ Jason Sheehan, M.D., Ph.D., ${ }^{4}$ \\ William Friedman, M.D., ${ }^{5}$ and Douglas KondziolKa, M.D. ${ }^{1}$
}

\begin{abstract}
${ }^{1}$ Department of Neurological Surgery, University of Pittsburgh, Pennsylvania; ${ }^{2}$ Radiosurgery Program, Yale University School of Medicine, New Haven, Connecticut; ${ }^{3}$ Department of Neurosurgery, Stanford University, Palo Alto, California; ${ }^{4}$ Department of Neurological Surgery and Gamma Knife Center, University of Virginia Health System, Charlottesville, Virginia; and ${ }^{5}$ Department of Neurosurgery, University of Florida, Gainesville, Florida
\end{abstract}

The field of stereotactic radiosurgery (SRS) was established by Lars Leksell in the 1950s as an adjunct to treatment of deep brain lesions otherwise not accessible to more conventional neurosurgery. Initially the focus of SRS was generation of functional lesions. Over time the procedure evolved and its focus expanded so that SRS now includes treatment of benign neoplastic or vascular lesions such as arteriovenous malformations (AVMs), meningiomas, and vestibular schwannomas. Since the mid1990 s, SRS has achieved a prominent role in the management of both brain and spinal benign and malignant tumors. ${ }^{2}$

When the focus of neurosurgical intervention was single benign lesions, the neurosurgeon was the primary decision maker in offering SRS as part of an armamentarium of lesion management options. Proper application of the stereotactic head frame and a sound understanding of the anatomical and physiological implications of adjacent brain areas were paramount in ensuring delivery of high-quality ongoing care for these patients.

Before the development of radiosurgical technologies that facilitate treatment of multiple tumors in 1 to 5 SRS sessions, most patients with metastatic brain cancer received whole brain radiation therapy as their only treatment option. Today treatment of brain and spine metastases is the most common indication for SRS. With this dramatic shift in the focus of SRS, the relative roles of the radiation oncologist, medical physicist, and neurosurgeon now vary considerably. Current spine and body radiosurgical procedures require increasingly complex linear accelerator (LINAC)-based technologies that are not included in the training of most neurological surgeons. Neurosurgical training includes acquisition of critical knowledge of neurological anatomy and function as well as alternative neurosurgical options and their outcomes. To date, radiosurgical treatment has achieved widespread implementation because of careful patient selection, optimal treatment planning, and dose delivery based on direct participation by a trained and responsible neurosurgeon partnering with a trained radiation oncologist. Loss of neurosurgery leadership in this process puts the radiosurgery field and, potentially, patients at risk.

According to data obtained from Elekta $\mathrm{AB}$, the manufacturer of Gamma Knife SRS technology, more than 700,000 patients underwent brain SRS worldwide during the last 33 years. The total number of LINAC SRS procedures remains unknown. The market research firm IMV estimates that 32,335 patients in the US underwent cranial LINAC SRS or Gamma Knife surgery (GKS) in 2009 and that the average increase in patient volume is $10 \%$ per year. The Center for Medicare and Medicaid Services reports that in 2010 the primary GKS hospital billing code, 77371, was billed 5,929 times, and the primary LINAC SRS billing code, G0339, was billed 9,337 times. The staged LINAC SRS billing code, G0340 (which represents image-guided robotic LINAC-based SRS, second through fifth sessions), was billed 22,193 times in the same year. Elekta, the Gamma Knife manufacturer, notes that 15,000 patients in the US underwent GKS in 2011. Despite this, current US neurosurgery residents participate in the delivery of less than $5 \%$ of radiosurgical procedures, even though most US teaching hospitals with more than 400 beds have SRS technologies.

In March 2012, we asked Katie O. Orrico, J.D., of the American Association of Neurological SurgeonsCongress of Neurological Surgeons (AANS-CNS) Washington Committee to review the number of SRS Current Procedural Terminology (CPT) codes submitted for payment to the Center for Medicaid and Medicare Services between 1993 and 2011 and to compare these numbers to the number of CPT codes submitted for craniotomy for tumor, not meningioma within the same interval. The data she provided are shown in Fig. 1 upper. In 2003 the number of CPT codes for radiosurgery (then 61793) that were submitted exceeded the number of codes submitted for craniotomy for tumor, not meningioma (Fig. 1 lower).

There are currently no clear program requirements for the training of neurosurgical residents in SRS. Relatively few programs offer dedicated "hands-on" experi- 

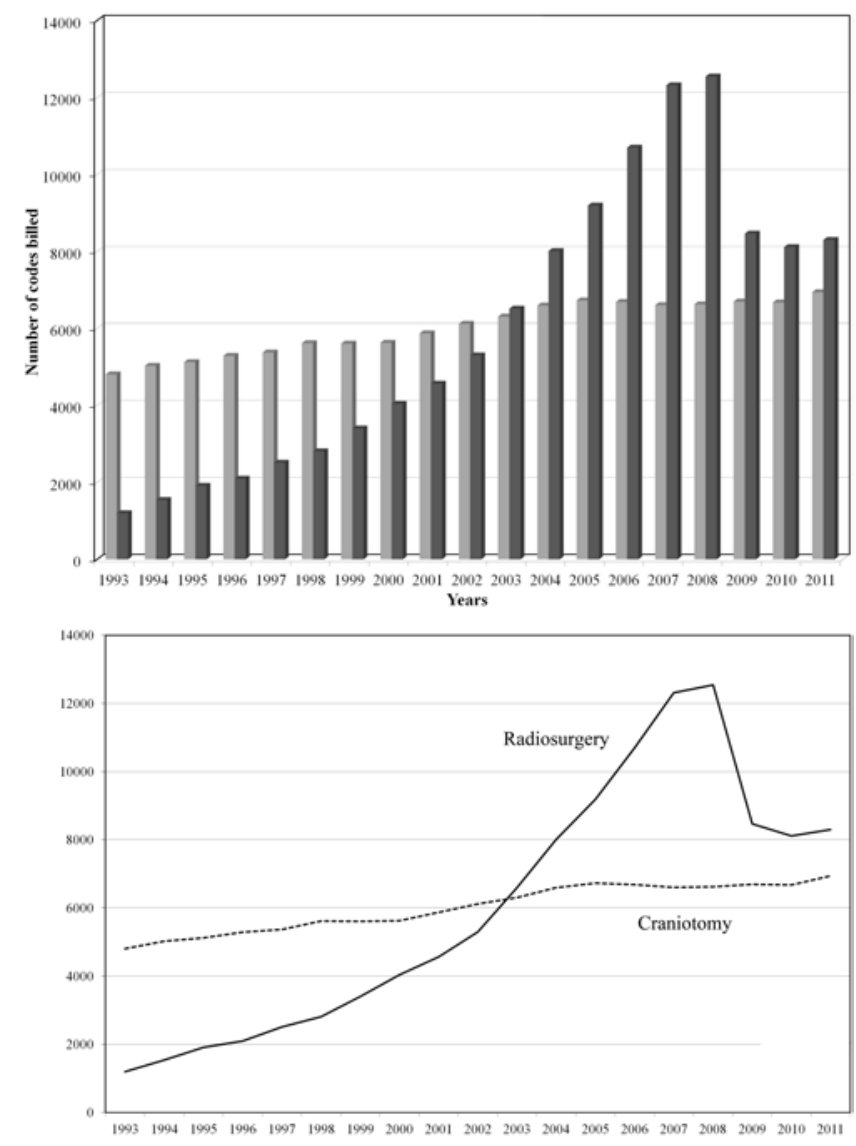

Fig. 1. Comparison of Center for Medicaid and Medicare Services CPT billing codes submitted for stereotactic radiosurgery and billing codes submitted for craniotomy for tumor, not meningioma, from 1993 to 2011. Upper: Between 1993 and 2008, surgeons submitted code 61793 for each SRS session. Centers where multisession SRSs were performed billed code 61793 for each session per patient. After the revision of the CPT codes in 1998, surgeons submitted one of two primary billing codes for SRS: 61796 for simple or 61798 for complex. The code submitted represented each treated lesion rather than each session. Black bars indicate codes for SRS; gray bars represent codes for craniotomy for tumor, not meningioma. Lower: The number of submitted billing codes for SRS $(61793,61796$, or 61798 [solid line]) surpassed the number of billing codes submitted for craniotomy for tumor, not meningioma (61510 [dashed line]) in 2003.

ence in SRS. While there are no "mandated" number of SRS cases, SRS experience in neurosurgery programs can be tracked using the Accreditation Council for Graduate Medical Education (ACGME) Accreditation Data System software that residents use to report their neurosurgery case loads. The US national resident experience in radiosurgery in 2011 included an average of 3 AVMs, 25 tumors, 5 functional neurosurgery cases, 3 spine cases, and 1 pediatric SRS case. The ACGME's Residency Review Committee for Neurosurgery recently defined the minimum numbers of cases required for residents in training as 10 SRS cases and 60 craniotomies for tumors during the 7 -year neurosurgery residency. ${ }^{1}$

Current requirements in radiation oncology training (effective January 2009, as cited in the ACGME radiation oncology program requirements PV IV.A. 5a) include a minimum experience of 10 intracranial cases and 5 spine and body cases during residency. It is therefore conceivable that teams of untrained neurosurgeons and radiation oncologists could be partnering to perform SRS procedures based on a minimal knowledge of the indications, methodologies, and potential risks of the procedure.

For the field of neurosurgery to remain an equal partner in the provision of SRS care, it is necessary that we provide adequate SRS training to our residents. Decreasing third party reimbursement of neurosurgeons, despite ongoing active participation in SRS, seems to reflect a public view that neurosurgical input into treatment delivery is of diminishing importance. As neurosurgeons, however, we must recognize that loss of neurosurgical input into SRS procedures will absolutely compromise the quality of ongoing care of the patient's neurological condition. It will also further distance our specialty from multidisciplinary care of patients with cancer.

As with all areas of neurosurgical training, experience in the use of SRS allows the resident to learn the advantages and limitations of various SRS technologies. While training does not need to be so comprehensive as to reach the level of expert user, neurosurgery residents need to be aware of the current indications, outcomes, and risks of SRS. Residents also need sufficient understanding of the procedure to provide adequate patient education about risks, benefits, and anticipated outcomes for particular clinical problems. Last, to continue to participate as coequal partners in the multidisciplinary SRS team, neurosurgeons need an adequate knowledge base to practice safe and effective SRS for diseases of the central nervous system.

The disease processes for which SRS is being routinely used in the US include the following:

\section{AVMs}

2. meningiomas

3. vestibular schwannomas

4. trigeminal neuralgia

5. pituitary adenomas

6. single and multiple metastases from cancer of the lung, breast, colon, kidney, ovary, prostate, and melanoma

7. recurrent ependymomas

8. selected glial neoplasms

9. miscellaneous skull-base tumors

10. paraspinal and spinal metastases

11. benign tumors of the spine such as schwannomas and chordomas

12. functional indications (epilepsy and movement and behavioral disorders)

The technologies that are available to deliver SRS include:

1. Leksell Gamma Knife ${ }^{\circledR}$ (Elekta AB)

2. LINAC-based technologies including but not limited to the Cyberknife ${ }^{\circledR}$ (Accurray Inc.), Synergy ${ }^{\circledR}$ (Elekta AB), Trilogy ${ }^{\circledR}$ and TrueBeam ${ }^{\mathrm{TM}}$ (Varian Medical System), XKnife ${ }^{\circledR}$ (Integra LifeSciences Corp.), and Novalis ${ }^{\circledR}$ (Brainlab)

3. proton beam therapy

It is important therefore for the neurosurgical resident to learn the following:

1. criteria for the safe use of SRS in each of the aforementioned diagnoses 
2. absolute and relative contraindications for using SRS in each of those diagnoses

3. possibilities of multidisciplinary approaches to the treatment of all of those diagnoses, particularly for minimizing morbidity and maximizing effectiveness of treatment.

4. recognition and management of acute and delayed possible complications of SRS

5. pitfalls in the delivery of SRS

6. dose-volume guidelines by lesion size, pathological characteristics, and neuroanatomical location

7. less common pathological disorders that are treatable by SRS

8. options for head or body immobilization or lesion localization

9. imaging interpretation after SRS during monitoring of radiosurgical effectiveness

10. principles and the practical use of various SRS delivery technology options delivery

11. use of imaging in the targeting of SRS treatment

12. use of SRS planning systems, image fusion, and the fallibilities of each system option

13. advantages and limitations of each technology

14. indications, methodologies, and outcomes of alternative radiation treatment options including standard fractionated external beam radiation therapy, imageguided radiation therapy, intensity-modulated radiation therapy, and multisession SRS procedures

15. basic medical physics, radiobiology, and quality assurance measures used to validate treatment planning and delivery

16. roles of additional members of the SRS team (including nurses, radiation therapists, medical physicists, dosimetrists, data managers, and administrative support personnel required for program quality

17. areas of current research, including both basic science and clinical research in the field of SRS, so that trainees can discuss controversies in current SRS practice

18. appropriate coding of intracranial and spinal SRS procedures

It is recognized that there is significant variability in the availability of SRS training opportunities across residency programs. Currently, selected centers with extensive radiosurgical experience include junior resident participation in patient preparation and dose planning, online radiosurgery courses, and focused rotations lasting 3-4 months. Senior residents may obtain additional elective experience as they prepare to enter practice and realize their need for additional training in SRS. Case loads for intracranial and spinal SRS should be documented. The case experience should be varied to include vascular malformations as well as benign and malignant neoplasms. To facilitate training in residencies in which subspecialty training opportunities are difficult to obtain, a model that was previously created for pediatric neurosurgery training could be used for developing alternate sites of SRS training. Options for the national education of neurosurgery residents in SRS could include 3- to 6-month intraresidency rotations at high-volume SRS centers of excellence. Additional options could include the following:

1. nationally offered courses or minifellowships at SRS centers of excellence as recognized by the American Board of Neurological Surgery (ABNS)

2. online training courses, potentially supplemented by an additional 3- to 4-week experience at an ABNSrecognized SRS center of excellence

3 . focused and practical courses during major national and international neurosurgery meetings (for example, annual meetings of the AANS or CNS)

4. industry-sponsored courses

5. multispecialty courses

6. specific technology-based academic meetings such as that offered by the Leksell Gamma Knife Society

7. AANS/American Society of Therapeutic Radiation Oncology SRS courses

We believe that the field of SRS will continue to grow in importance. It represents a unique paradigm shift in the management of a wide variety of cranial and now spinal disorders. It is also a unique collaboration among medical professionals. It is incumbent on our discipline to ensure that the next generation of neurological surgeons are trained and prepared to colead this SRS evolution. (http://thejns.org/doi/abs/10.3171/2012.6.GKS12838)

\section{Disclosure}

Dr. Lunsford holds stock in Elekta AB. Both Drs. Lunsford and Kondziolka are consultants to Elekta AB. Dr. Adler is an employee of and holds stock in Varian Medical Systems, Inc.

\section{Acknowledgments}

The authors are grateful to Katie O. Orrico, J.D., and the staff of the AANS-CNS Washington Committee for their assistance in obtaining the data we reviewed from the Center for Medicaid and Medicare Services.

\section{References}

1. Accreditation Council for Graduate Medical Education: Neurological Surgery. RRC News, April 2012 (http://www. acgme.org/acWebsite/RRC_160_News/Neuro_Surgery_ Newsletter_Apr12.pdf) [Accessed June 7, 2012]

2. Kondziolka D, Lunsford LD, Loeffler JS, Friedman WA: Radiosurgery and radiotherapy: Observations and clarifications. J Neurosurg 101:585-589, 2004

This paper was presented in part by Dr. Lunsford at the 16th International Meeting of the Leksell Gamma Knife Society in March 2012, in Sydney, Australia.

Please include this information when citing this paper: DOI: 10.3171/2012.6.GKS12838. 